\title{
Revisión y recomendaciones para el manejo de diarrea por Vibrio parahaemolyticus
}

\author{
INGRID HEITMANN G., LEONOR JOFRÉ M., \\ J. CARLOS HORMÁZABAL O., ANDREA OLEA N., \\ CLELIA VALLEBUONA S. y CLAUDIO VALDÉS H.
}

\section{Review and guidelines for treatment of diarrhea caused by Vibrio parahaemolyticus}

In Chile Vibrio parahaemolyticus has been detected in 3 gastroenteritis outbreaks since 1998. The most recent outbreak occurred during the summer of 2005, affecting over 10.000 people of whom one died. Affected individuals presented with one or more of the following symptoms: diarrhea, nausea, vomiting, abdominal pain and/or fever. Fecal white blood cells were detected in only $6 \%$ of patients. The predominant serotype in the 3 outbreaks was the pandemic O3:K6 strain. Diagnosis was confirmed by isolation and identification of $V$. parahaemolyticus in stool cultures and/or by establishing an epidemiological link. V. parahaemolyticus isolates were $100 \%$ susceptible to tetracycline, ciprofloxacin and chloramphenicol, and universally resistant to ampicillin. Due to the public health impact of the 2005 outbreak, the Ministry of Health called for a National Task Force mandated to review epidemiological, clinical and microbiological features of the outbreak and to propose management guidelines.

Key words: Vibrio; Vibrio parahaemolyticus; Foodborne disease; Seafood; Diarrhea; Diarrhea outbreak.

Palabras claves: Vibrio; Vibrio parahaemolyticus; Enfermedad transmitida por alimentos; Mariscos; Diarrea; Brote epidémico de diarrea.

\section{Introduccción}

Durante mucho tiempo el interés de los médicos clínicos se ha centrado en Vibrio cholerae, responsable de brotes de gran magnitud con una alta letalidad, especialmente en países en desarro$10^{1}$. En la última década han emergido otros Vibrio productores de enfermedades que, aunque generalmente de menor severidad, tienen la capacidad de producir importantes brotes epidémicos, como Vibrio parahaemolyticus ${ }^{2}$.

La infección por Vibrio es adquirida por la vía oral o por inoculación a través de piel no intacta ${ }^{3}$, afectando así al tracto digestivo, y produciendo ocasionalmente infecciones cutáneas y síndromes sépticos ${ }^{4,5}$.
El género Vibrio, de la familia Vibrionaceae, posee más de 48 especies, y su taxonomía está en constante revisión gracias a la incorporación de técnicas de biología molecular. Al menos 12 de ellas son patógenas para el hombre y varias son también patógenas para animales tanto vertebrados como invertebrados ${ }^{6}$ (Tabla 1).

El hábitat natural de $V$. parahaemolyticus está en las aguas marinas costeras, especialmente los estuarios, las que representan su reservorio. La población microbiana es afectada por los cambios en la temperatura, salinidad, disponibilidad de nutrientes y su asociación con animales marinos. La temperatura óptima para su desarrollo es entre 20 y $30^{\circ} \mathrm{C}$, la que permite una mayor concentración de bacterias; bajo $20^{\circ} \mathrm{C}$ disminuye

Instituto de Salud Pública de Chile, Santiago, Chile (IHG, JCHO).

Hospital Clínico Universidad de Chile, Santiago, Chile (LJM).

Departamento de Epidemiología, Ministerio de Salud, Chile (AON, CVS).

Hospital de Enfermedades Infecciosas Dr Lucio Córdova, Santiago, Chile (CVH).

Recibido: 31 marzo 2004

Aceptado: 5 abril 2005 
Tabla 1. Asociación de Vibrio patógenos con síndromes clínicos

\begin{tabular}{lccc}
\hline Vibrio sp & Diarrea & Sepsis & Infección de heridas \\
\hline V. cholerae 01 & +++ & no & + \\
V. cholerae no 01 & +++ & + & ++ \\
V. parahaemolyticus & +++ & + & ++ \\
V. vulnificus & ++ & +++ & +++ \\
V. fluvialis & ++ & + & + \\
V. alginolyticus & no & + & +++ \\
V. damsela & no & no & ++ \\
V. furnissii & + & no & no \\
V. hollisae & ++ & no & no \\
V. mimicus & ++ & no & ++ \\
V. metschnikovii & + & + & no \\
V. cincinatiensis & no & + & no \\
\hline
\end{tabular}

Adaptado de referencias ${ }^{1,2,6,47}$.

su densidad y a $10^{\circ} \mathrm{C}$ "desaparece" de la columna de agua, pero se mantiene en el sedimento desde donde reemerge cuando las condiciones son otra vez favorables (Ej. meses cálidos en regiones templadas) ${ }^{7}$. La salinidad requerida es entre 5 y $30 \%$ (bacterias halofílicas), concentración que puede variar dependiendo de la temperatura ambiental y disponibilidad de nutrientes. Se asocia a la presencia de plancton y moluscos bivalvos para sobrevivir en agua de mar, los que acumulan Vibrio durante el proceso de filtración y alimentación, alcanzando concentraciones hasta 100 veces superiores a las del agua. Puede colonizar la superficie o formar parte de la flora comensal de algunos peces ${ }^{8,3}$. En el medioambiente, V. parahaemolyticus es mayoritariamente no patógeno; usualmente menos del $1 \%$ de los aislados de origen ambiental (agua, mariscos) poseen la capacidad de producir infección entérica (presencia de mayores factores de virulencia) ${ }^{32,46}$.

Vibrio parahaemolyticus fue reconocido en el año 1950 como agente de enfermedades transmitidas por alimentos (ETA) en Japón, en un brote epidémico asociado al consumo de sardinas que afectó a 272 personas, de las cuales 20 fallecieron. Desde entonces ha adquirido un rol más protagónico como agente causal de infección gastrointestinal relacionada al consumo de mariscos crudos o insuficientemente cocidos. Se han reportado brotes epidémicos en diversas regiones como Asia, las Américas y Europa (España) ${ }^{8,9,10}$.

\section{Aspectos epidemiológicos}

Vibrio parahaemolyticus es un enteropatógeno emergente en Chile, que ha producido brotes epidémicos en clara asociación con el consumo de mariscos, especialmente moluscos bivalvos crudos o mal cocidos.

Antes de 1997 V. parahaemolyticus se encontraba en forma esporádica en nuestro país. Entre 1992 y 1997 el Instituto de Salud Pública de Chile (ISP), recibió solamente 30 aislados de muestras clínicas provenientes de los hospitales regionales para su identificación. Sin embargo, un brote epidémico ocurrido en Antofagasta entre noviembre de 1997 y marzo de 1998, periodo de gran actividad de la Corriente del Niño, afectó a 340 personas posicionando a $V$. parahaemolyticus como causa importante de ETA en nuestro país. En esa oportunidad $V$. parahaemolyticus fue detectado en almejas, cholgas y ostiones ${ }^{11}$. El segundo brote epidémico reportado en Chile ocurrió en el Servicio de Salud Llanquihue-ChiloéPalena (Llanchipal) de la $\mathrm{X}^{\mathrm{o}}$ Región, al sur del país, entre enero y marzo de 2004 y afectó a más de 1.500 personas, mayoritariamente adultos. En dicha oportunidad el ISP confirmó microbiológicamente los casos, detectando la presencia de $V$. parahaemolyticus con toxina TDH. Secundariamente se produjeron brotes epidémicos en otras regiones donde se consumían mariscos procedentes de Llanchipal, como la Región Metropolitana $(\mathrm{RM}), \mathrm{V}^{\mathrm{o}}, \mathrm{VIII}^{\mathrm{o}}$ y $\mathrm{IX}^{\mathrm{o}}$ regiones. Un tercer brote epidémico, de mayor magnitud que los anteriores, se inició en el verano del 2005. El día 4 de enero comenzaron a aparecer casos de gastroenteritis compatibles con un cuadro de intoxicación por $V$. parahaemolyticus en la $\mathrm{X}^{\mathrm{o}}$ Región, extendiéndose a la $\mathrm{V}^{\mathrm{o}}$ Región los primeros días de febrero. Posteriormente, aparecieron 
casos en la RM y resto del país, con excepción de las regiones II $^{\circ}$ y $\mathrm{III}^{\circ}$. El total acumulado al 23 de marzo asciende a 10.491 casos, 789 confirmados por el ISP, y el resto por nexo epidemiológico (Figura 1). El 35\% del total de casos corresponde a la $\mathrm{X}^{\mathrm{o}}$ Región, afectando ligeramente más a hombres $(52 \%)$ y mayoritariamente a adultos (mediana de edad de 39 años).

Respecto a la gravedad, medida en términos de hospitalización, ésta fue indicada en $0,6 \%$ de los casos (59). En la VI Región se debió hospitalizar a 1,8\% del total de los casos, mientras que en la $\mathrm{X}^{\mathrm{o}}$ Región, que concentra el $35 \%$ de los casos totales del país, esta cifra fue de 1\% (33 casos). De acuerdo al análisis realizado por la $\mathrm{X}^{\mathrm{o}}$ Región, los hospitalizados correspondieron a adultos, con una mediana de edad de 39 años (rango: 14-74 años), sin diferencias por sexo. A la fecha, sólo una muerte ha sido atribuible al brote epidémico (Tabla 2).

\section{Aspectos microbiológicos}

Morfología y cultivo. Vibrio parahaemolyticus es un bacilo gramnegativo, levemente curvo, aerobio facultativo, halofílico, oxidasa positiva, fermentador de glucosa, pero no de sacarosa, y ureasa variable. Requiere de medios selectivos para su desarrollo, con una concentración de $\mathrm{NaCl}$ de $1 \%{ }^{12,13}$. En medio TCBS las colonias se observan de color verde, a diferencia de $V$. cholerae que es de color amarillo.

Patogenicidad. La toxina termoestable directa $(\mathrm{TDH})$ es el factor de virulencia más importante en el mecanismo de producción de la diarrea $^{13-19}$. La TDH es una proteína con actividad hemolítica sobre una variada gama de eritrocitos (fenómeno de Kanagawa); esta toxina posee varias propiedades entre las que destacan: citotoxicidad, aumento de la permeabilidad vascular y acumulación de líquido en el asa de ileon en el modelo experimental en conejos. El mecanismo patogénico es la alteración del flujo iónico de las células intestinales, el que desencadena una diarrea secretora. La toxina TDH es codificada por un gran número de genes $t d h$, los que han sido secuenciados, evidenciándose una estrecha relación genética entre ellos (97\% de similitud). Otro factor importante en la producción de diarrea es la presencia de la toxina hemolisina relacionada (TRH), que es codificada por los genes $t r h$, genéticamente relacionado a $t d h$, con $68,6 \%$ de similitud genética ${ }^{13,16,19}$. Esta toxina fue inicialmente determinada en cepas provenientes de casos de gastroenteritis que no presentaban el fenómeno hemolítico de Kanagawa. Al igual que TDH, TRH produce acumulación de líquido en el modelo experimental de asa ileal y presenta actividad citotóxica en una variedad de tejidos. Además de los anteriores, $V$. parahaemolyticus requiere de otros factores para causar enfermedad, como una variedad de pili, hemaglutininas (hemaglutinina manosa sensitiva, mannose sensitive hemagglutinin-MSHA), factores de colonización y capacidad de invasión celular ${ }^{13,20}$.

Tipificación. Existen diversos métodos de tipificación para $V$. parahaemolyticus, tanto fenotípicos como moleculares. Dentro de los primeros destaca la serotipificación de lipopolisacáridos somáticos $(\mathrm{O})$ y polisacáridos capsulares $(\mathrm{K})$. El esquema antigénico de tipificación fue diseñado inicialmente por Sakazaki en Japón en 1963, y complementado posteriormente por otros inves-

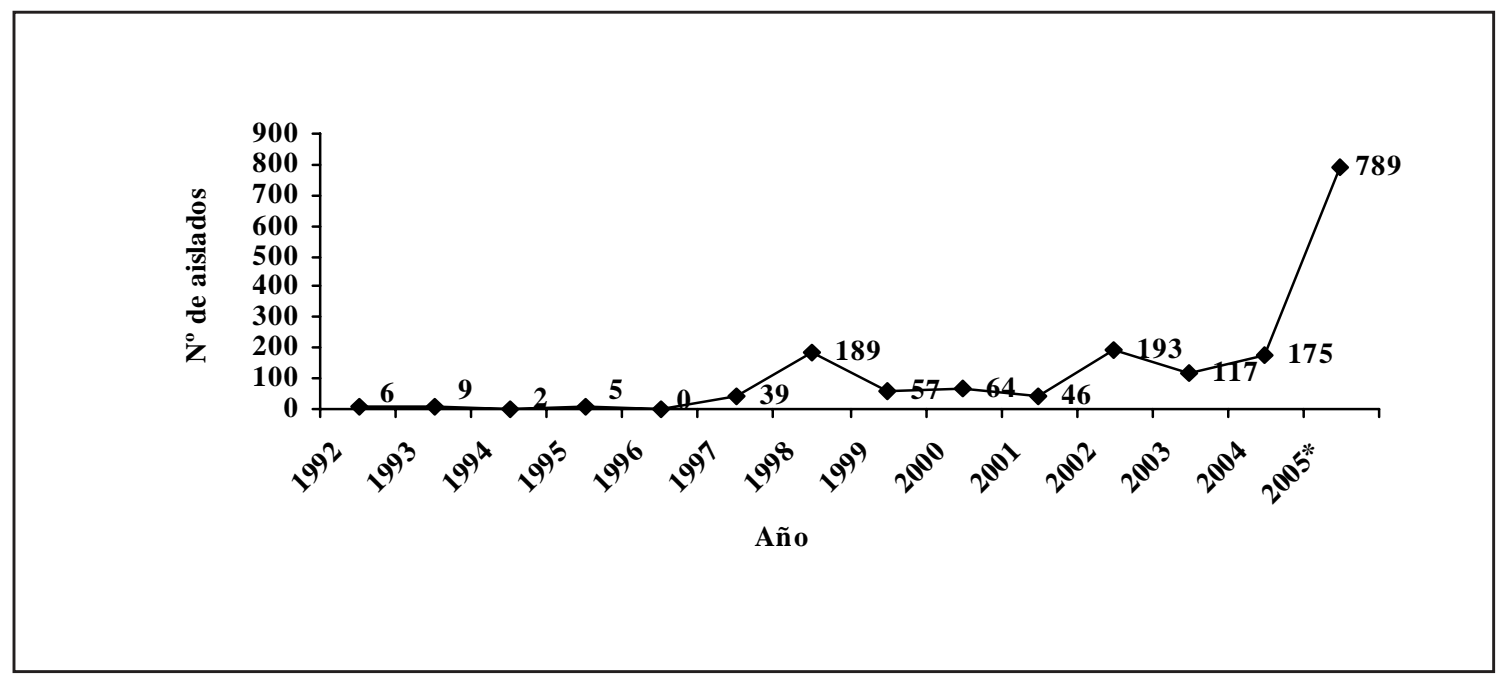

Figura 1. Cepas de Vibrio parahaemolyticus de origen clínico. Laboratorio de Referencia ISP, período 1992- marzo 2005. 
Tabla 2. Brote de gastroenteritis por Vibrio parahaemolyticus Hospitalización y letalidad. Chile, enero-marzo 2005

\begin{tabular}{|c|c|c|c|c|c|c|c|}
\hline \multicolumn{2}{|c|}{ Región } & \multicolumn{2}{|c|}{$\begin{array}{c}\text { Casos } \\
\text { (al 22/03/2005) }\end{array}$} & \multicolumn{2}{|c|}{ Hospitalizados } & \multicolumn{2}{|c|}{ Fallecidos } \\
\hline & & $\mathrm{n}$ & $\%$ & $\mathbf{n}$ & $\%$ & $\mathbf{n}$ & $\%$ \\
\hline I & Tarapacá & 8 & 0,1 & 1 & 12,5 & 0 & 0,0 \\
\hline II & Antofagasta & 0 & 0,0 & 0 & 0,0 & 0 & 0,0 \\
\hline III & Atacama & 0 & 0,0 & 0 & 0,0 & 0 & 0,0 \\
\hline IV & Coquimbo & 354 & 0,0 & 0 & 0,0 & 0 & 0,0 \\
\hline V & Aconcagua & 1.271 & 12,1 & 2 & 0,2 & 0 & 0,0 \\
\hline VI & O'Higgins & 341 & 3,3 & 6 & 1,8 & 0 & 0,0 \\
\hline VII & Maule & 839 & 8,0 & 0 & 0,0 & 0 & 0,0 \\
\hline VIII & Bío-Bío & 2.149 & 20,5 & 15 & 0,7 & 0 & 0,0 \\
\hline IX & Araucanía & 262 & 2,5 & 1 & 0,4 & 0 & 0,0 \\
\hline $\mathrm{X}$ & Los Lagos & 3.693 & 35,2 & 33 & 0,9 & 1 & 0,3 \\
\hline XI & Aysén & 2 & 0,0 & 0 & 0,0 & 0 & 0,0 \\
\hline XII & Magallanes & 2 & 0,0 & 0 & 0,0 & 0 & 0,0 \\
\hline \multirow[t]{2}{*}{$\mathrm{RM}$} & & 1.570 & 15,0 & 1 & 0,1 & 0 & 0,0 \\
\hline & País & 10.491 & 100 & 59 & 0,6 & 1 & 0,1 \\
\hline
\end{tabular}

Elaborado por Dpto. Epidemiología Ministerio de Salud de Chile.

Fuente: Notificaciones de SREMIS de Salud de Chile.

tigadores ${ }^{21,22}$. Actualmente existen kits comerciales que reconocen 13 grupos $\mathrm{O}$ y 71 tipos K. En relación a los métodos moleculares, existe una gran variedad, destacando la determinación de genes $t d h$ y $t r h$ por RPC, de gran utilidad para diferenciar cepas patógenas de las no patógenas, ya que ambos genes representan los mayores factores de virulencia de $V$. parahaemolyticus ${ }^{18,23}$. Para la determinación de genotipos se puede realizar electroforesis de campo pulsado (PFGE) $)^{24-}$ ${ }^{27}$, ribotipificación y diversas técnicas basadas en amplificación como RPC con partidores arbitrarios (AP-PCR), amplificación de elementos palindrómicos extragénicos repetitivos (REPPCR), amplificación de secuencias intergénicas de consenso repetitivas (ERIC-PCR) ${ }^{26-31}$, etc. La PFGE representa la técnica de tipificación genética de elección por su poder discriminatorio, estandarización y capacidad de almacenamiento de los distintos patrones genéticos en bases de datos.

Epidemiología. Hasta el año 1995 las infecciones producidas por $V$. parahaemolyticus estaban asociadas a varios serotipos, salvo casos reportados en la costa oeste de E.U.A. y México, en que el serotipo predominante era $\mathrm{O} 4: \mathrm{K} 12^{32}$. En febrero de 1996 en Calcuta, India, se detectó por primera vez un nuevo clon de $V$. parahaemolyticus perteneciente al serotipo O3:K6, TDH positivo, TRH negativo, siendo responsable de un gran aumento de los casos de enfermedad diarreica en esa zona ${ }^{29}$. Posteriormente este clon pandémi$\mathrm{Co}^{25,28,29-34}$ ha sido el responsable del aumento de casos en otras regiones de Asia, llegando incluso a Norteamérica y finalmente a Chile, donde se ha detectado su presencia desde el año 1998; desde entonces ha sido el responsable de todos los grandes brotes ocurridos ${ }^{35}$ (comunicación verbal ISP). El fundamento de la expansión de este clon permanece sin dilucidar, puesto que su nivel de producción de TDH y su susceptibilidad antimicrobiana no es distinta a la de otras cepas patógenas de $V$. parahaemolyticus. Se especula que su capacidad de persistir en el medioambiente o su habilidad en producir infección sean las bases de su éxito como patógeno ${ }^{36}$.

Otros serotipos como O4:K68, O1: KNT (no tipificable), O1:K25, O1:K41, O4:K12, han emergido en diversas regiones del mundo, determinándose por variados estudios moleculares su asociación genética con el clon pandémico $\mathrm{O} 3: \mathrm{K}^{37,38,9}$.

Susceptibilidad in vitro. En relación a susceptibilidad antimicrobiana, no hay estándares de interpretación para $V$. parahaemolyticus (guías NCCLS-CLSI 2005). Usando los estándares de interpretación para $V$. cholerae y Enterobacteriaceae $^{12,13}, V$. parahaemolyticus es sensible a tetraciclina, doxiciclina, furazolidona, cotrimoxazol, cloranfenicol, cefalosporinas de tercera 
generación, aminoglucósidos y quinolonas. La resistencia a colistin y ampicilina (esta segunda mediada por $\beta$ lactamasas), es variable. Las cepas aisladas en nuestro país son $100 \%$ sensibles a cloranfenicol y ceftriaxona, $98 \%$ a tetraciclina, $97 \%$ a ácido nalidíxico, $90 \%$ a ciprofloxacina y $30,8 \%$ a aminoglucósidos. No se han detectado cepas sensibles a ampicilina (Tabla 3).

Vigilancia. En Chile V. parahaemolyticus es objeto de vigilancia de laboratorio desde el año 2000, cuando entró en vigencia el Reglamento sobre Notificación de Enfermedades Transmisibles de Declaración Obligatoria $\mathrm{N}^{\circ} 712$ del Ministerio de Salud, por el cual los laboratorios clínicos públicos y privados que identifiquen este agente están obligados a notificarlo al Instituto de Salud Pública de Chile. En la Figura 2 se muestra el total de aislados de $V$. parahaemolyticus confirmados por el laboratorio de referencia en el brote epidémico del año 2005.El envío de las cepas debe realizarse en tubos de agar Mueller Hinton o agar soya tripticasa, debidamente sellados, rotulados y a temperatura ambiente. Es importante no enviar en medios que contengan carbohidratos, porque los subproductos ácidos del metabolismo disminuyen su viabilidad. Las cepas deben ir acompañadas del formulario $a d$ hoc de la Sección Bacteriología del Instituto de Salud Pública de Chile. Al enviar la cepa se considera automáticamente que está notificada pero, en aquellos casos en que por algún motivo no pudiera enviarse la cepa, la notificación debe realizarse igualmente en el formulario antes mencionado (Figura 3).

Tabla 3. Susceptibilidad in vitro de V.parahaemolyticus (n: 99). Instituto de Salud Pública. 2005

\begin{tabular}{|c|c|c|c|c|c|c|}
\hline \multirow{2}{*}{$\begin{array}{l}\text { Antimicrobiano } \\
\text { Ampicilina* }\end{array}$} & \multicolumn{2}{|c|}{ Resistente } & \multicolumn{2}{|c|}{$\begin{array}{l}\text { Interpretación } \\
\text { Intermedio }\end{array}$} & \multicolumn{2}{|c|}{ Sensible } \\
\hline & 61 & $(61,6 \%)$ & 38 & $(38,4 \%)$ & 0 & \\
\hline Tetraciclina* & & $(1 \%)$ & 0 & & 98 & $(99 \%)$ \\
\hline Gentamicina** & 1 & $(1 \%)$ & 61 & $(61,6 \%)$ & 37 & $(37,4 \%)$ \\
\hline Ciprofloxacina** & 0 & & 7 & $(7,1 \%)$ & 92 & $(92,9 \%)$ \\
\hline Cotrimoxazol* & 0 & & 1 & $(1 \%)$ & 97 & $(99 \%)$ \\
\hline A. Nalidíxico** & 2 & $(2 \%)$ & 0 & & 97 & $(98 \%)$ \\
\hline Cloranfenicol* & 0 & & 0 & & 97 & $(100 \%)$ \\
\hline Ceftriaxona** & 0 & & 0 & & 99 & $(100 \%)$ \\
\hline
\end{tabular}

* Lectura tabla NCCLS de Vibrio cholerae.

** Lectura tabla NCCLS Enterobacterias.

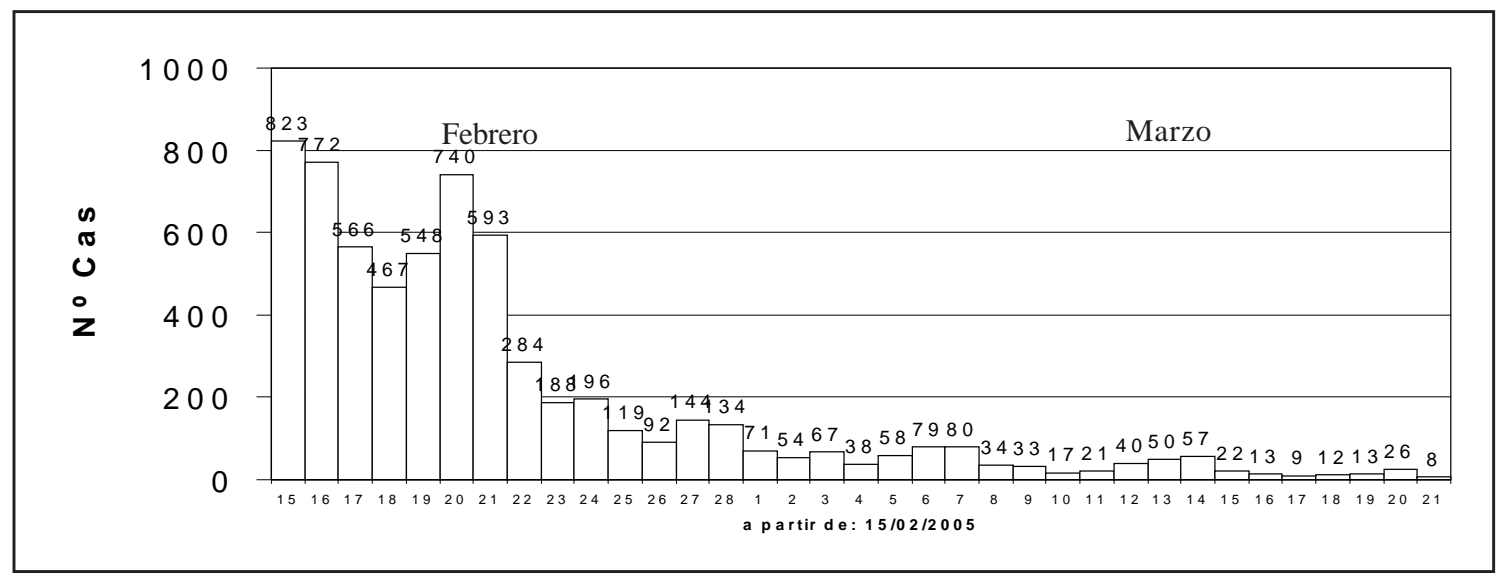

Figura 2. Casos de gastroenteritis por Vibrio parahaemolyticus. Total país*. No incluye acumuladas hasta el 14 de febrero. 


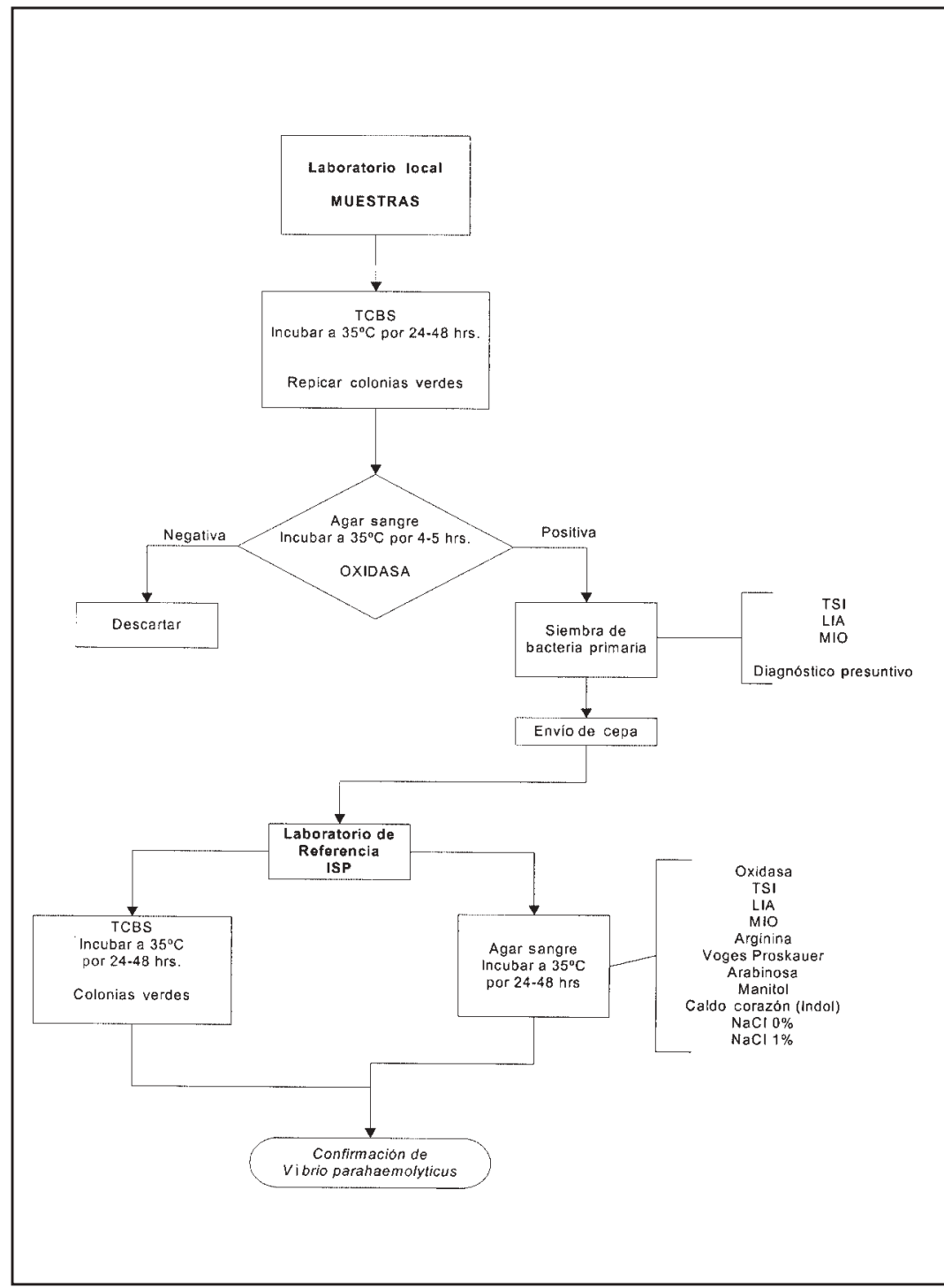

Figura 3. Diagnóstico presuntivo de Vibrio parahaemolyticus. Laboratorio Referencia de Vibrios, Sección Bacteriología, ISP 2005.

\section{Características clínicas}

En general la infección por $V$. parahaemolyticus produce un cuadro de gastroenteritis aguda pero también se ha aislado a partir de infección de heridas y septicemias. En E.U.A en una serie de pacientes con infección por este agente, 59\% presentó diarrea, 34\% infección de heridas, $5 \%$ síndrome séptico y $2 \%$ tuvieron otras manifestaciones ${ }^{9}$. En Chile hasta el momento no se han reportado infecciones extraintestinales. El análisis de los síntomas y signos del último brote se muestra en la Tabla 4.

La dosis infectante requerida es de $10^{5}$ a $10^{7}$ bacterias y el período de incubación promedio es de $17 \mathrm{hrs}$ con un rango de 4 a $90 \mathrm{hrs}{ }^{3,9}$.
Tabla 4. Brote de gastroenteritis por Vibrio parahaemolyticus. Principales síntomas y signos. Chile, enero - febrero 2005

\begin{tabular}{lc}
\hline Síntomas y signos & \% de frecuencia \\
\hline Dolor abdominal & 90 \\
Náuseas y vómitos & 89 \\
Diarrea secretoria & 80 \\
Fiebre & 77 \\
Deshidratación & 38 \\
Parestesias & 16 \\
Calambres & 16 \\
Diarrea con sangre & 6 \\
Cefalea & 4 \\
\hline
\end{tabular}

Elaborado por Departamento de Epidemiología, Ministerio de Salud de Chile. Fuente: SEREMI X ${ }^{\circ}$ Región ( $\mathrm{n}=502$ acumulados al día 12 de febrero 2005). 
El cuadro diarreico que produce $V$. parahaemolyticus es generalmente leve y de evolución autolimitada, con una duración promedio de tres días (rango de 12 horas a 12 días). Las manifestaciones clínicas son dolor abdominal (89\%), náuseas $(76 \%)$, vómitos $(55 \%)$ y fiebre $(52 \%)^{9}$. En un tercio de los casos se puede encontrar leucocitos fecales positivos, manifestación más frecuente en India y Bangladesh ${ }^{1}$. Se ha descrito también cuadros "tipo cólera", especialmente en Indonesia 39 .

Desarrollan un cuadro de mayor gravedad los pacientes con hepatopatías de diverso origen, alcohólicos, diabéticos, individuos con aclorhidria, inmunocomprometidos, mujeres embarazadas y pacientes con edades extremas ${ }^{6,9,40,41}$.

En pacientes que han sido sometidos a trasplante de órganos se ha descrito diarrea prolongada con mala-absorción, por lo que es muy importante la prevención en esta población de riesgo ${ }^{42,43}$.

\section{Tratamiento}

El manejo es fundamentalmente de rehidratación y corrección de los trastornos hidroelectrolíticos y ácido-base ${ }^{1,9}$. No debe usarse antiespasmódicos ni inhibidores de la motilidad intestinal, no hay estudios con el uso de probióticos en infecciones por este agente. Tampoco se ha demostrado que el uso de antimicrobianos abrevie el curso de la infección; sin embargo, se recomienda su empleo cuando la diarrea excede más de 5 días, en infecciones extraintestinales y en pacientes con co-morbilidad ${ }^{2,9,40-44}$.

El fármaco recomendado en estos casos es tetraciclina o doxiciclina a partir de los 9 años de edad o una fluoroquinolona, por un período no menor de 5 días. En pacientes pediátricos pueden usarse cloranfenicol o cotrimoxazol, dependiendo de la susceptibilidad antimicrobiana, o ciprofloxacina en caso de no observarse respuesta al esquema inicial. En infecciones cutáneas o septicemia por este agente, se recomienda tratar agresivamente con antimicrobianos asociados, como cefalosporinas de tercera generación + doxiciclina o aminoglucósidos, o quinolonas + aminoglucósidos, esquema similar al empleado en infecciones causadas por Vibrio vulnificus ${ }^{1,40-45}$. El uso de tetraciclina está avalado en los estudios iniciales por su acción en la síntesis proteica, lo que disminuye la liberación de la toxina TDH.

Como una manera de orientar al médico que recibe y maneja estos pacientes, se diseñó una pauta que considera los factores de riesgo y presentación clínica. A cada uno de estos factores se les asigna un puntaje y de acuerdo a esto se sugiere el inicio o no de tratamiento específico (Tablas 5 y 6 ).

\section{Prevención}

Dado que en la actualidad V. parahaemolyticus se encuentra presente en los mariscos de nuestro litoral, es necesario que la población tome las siguientes medidas:

- Consumir siempre los mariscos cocidos.

- Hervir los mariscos durante al menos 5 minutos antes de consumirlos.

- Lavar muy bien las manos al cocinar.

- Mantener los mariscos refrigerados.

- Evitar que se produzca contaminación cruzada:

- Manipular separadamente los mariscos crudos del resto de los alimentos.

- Después de manipular los mariscos crudos, lavar las manos.

- Limpiar los mesones y cubiertas donde se preparan los alimentos.

- Consumir mariscos sólo en lugares públicos autorizados.

- No olvidar el riesgo de contaminación cruzada entre mariscos contaminados y pescados, siendo recomendable extender sobre estos últimos las medidas antes mencionadas.

\section{Resumen}

A contar del año 1998 se han presentado en Chile tres brotes epidémicos por Vibrio parahaemolyticus, el último de ellos durante el verano del 2005, que afectó a más de 10.000 personas. Los afectados presentaron un cuadro clínico caracterizado por diarrea, náuseas, vómitos, dolor abdominal y fiebre; $6 \%$ de los casos tuvo leucocitos fecales positivos y un paciente falleció. La cepa predominante en los tres brotes ha sido la pandémica O3: K6. El diagnóstico de $V$. parahaemolyticus se realizó con la confirmación microbiológica de las cepas y tipificación o por asociación epidemiológica. Las cepas fueron susceptibles in vitro a tetraciclina, cefalosporinas de tercera generación, quinolonas y cloranfenicol no observándose susceptibilidad a ampicilina. Todos los casos se asociaron al consumo de mariscos crudos o insuficientemente cocidos. Por la repercusión de este brote, el Ministerio de Salud impulsó la formación de una comisión multidisciplinaria para actualizar los aspectos epidemiológicos, clínicos y microbiológicos, y elaborar una guía de recomendaciones en el manejo de esta infección. 
Tabla 5. Factores de riesgo para gravedad en infección por $V$. parahaemolyticus y asignación de puntaje

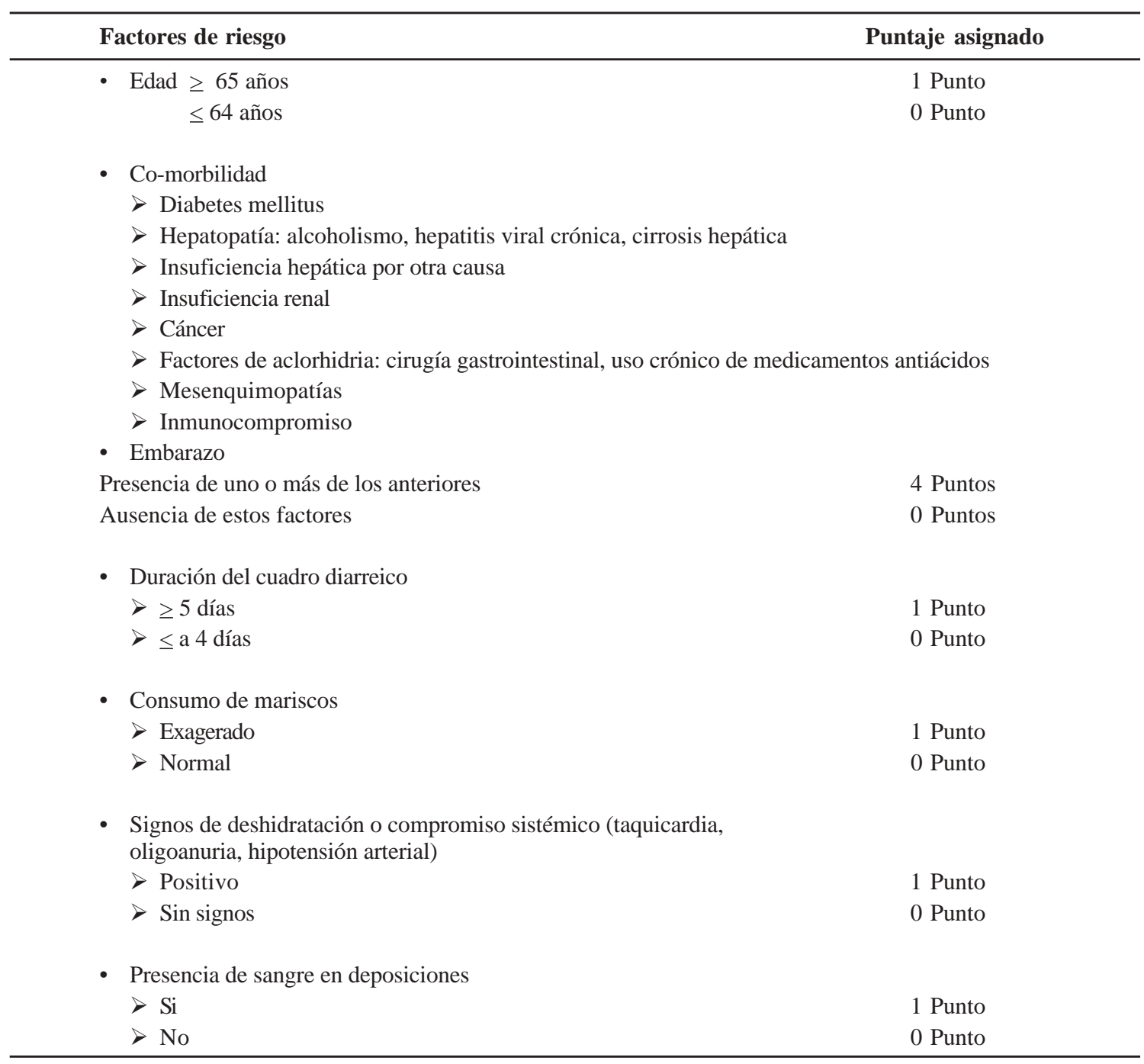

Tabla 6. Aplicación del puntaje

\begin{tabular}{lll}
\hline Puntaje & Gravedad & Tratamiento \\
\hline 4 ó más puntos & Grave & Hospitalización, \\
& & Hidratación parenteral \\
& & Indicar antibacterianos \\
& Moderada & \\
& & Tratamiento ambulatorio, sintomático, con rehidratación \\
& & oral o endovenosa en unidad de emergencia \\
& & Citar a control en 24 horas. \\
Menos de 2 puntos & Leve & Tratamiento ambulatorio, sintomático, hidratación oral, \\
& & Dieta sin residuos, \\
& & Control en 24 horas si agrava o no hay mejoría. \\
\hline
\end{tabular}


Agradecimientos: A Jorge Segovia y M. Isabel Campos por su participación en preparación de la pauta de manejo y a la Sección de Bacteriología del Instituto de Salud Pública de Chile.

\section{Bibliografía}

1.- Morris J G. Cholera and other types of vibriosis: a story of human pandemics and oysters on the half shell. Clin Infect Dis 2003; 37: 272-80.

2.- Tantillo G M, Fontanarosa M, Di Pinto A, Musti M. Updated perspectives on emerging vibrios associated with human infections. Lett Appl Microbiol 2004; 39: $117-26$.

3.- Draft risk assessment on the Public Health impact of Vibrio parahaemolyticus in raw molluscan shellfish, Center for Food Safety and Applied Nutrition Food and Drug Administration U.S. Department of health and Human Services, Dec. 2000.

4.- Collier D N. Cutaneous infections from coastal and marine bacteria. Dermatologic Therapy 2002; 15:1-9.

5.- Hlady W G, Klontz K C. The epidemiology of Vibrio infections in Florida, 1981-1993. J Infect Dis 1996; 173: $1176-83$.

6.- Morris J G, Black R. Cholera and other vibrioses in the United States. N Engl J Med 1985; 312: 343-50.

7.- Hervio-Heath D, Colwell R R, Derrien A, RobertPillot A, Fournier J M, Pommepuy M. Ocurrence of pathogenic vibrios in the coastal areas of France. J Appl Microbiol 2002; 92: 1123-35.

8.- Martínez-Urtaza J, Lozano-León A, DePaola A, Ishibashi M, Shimada K, Nishibuchi M, et al. Characterization of pathogenic Vibrio parahaemolyticus isolated from clinical sources in Spain and comparison with Asian and North American pandemic isolates. J Clin Microbiol 2004;42: 4672-8.

9.- Daniels N A, Mackinnon L, Bishop R, Altekruse S, Ray B, Hammond R M, et al. Vibrio parahaemolyticus infections in the United States, 1973-1998. J Infect Dis 2000; 181: 1661-6.

10.- Alam M J, Miyoshi S, Shinoda S. Studies on pathogenic Vibrio parahaemolyticus during a warm weather season in the Seto Island Sea, Japan. Environ Microbiol 2003; 5: 706-10

11.- Córdova J L, Astorga J, Silva W, Riquelme C. Characterization by PCR of Vibrio parahaemolyticus isolates collected during 1997-1998 Chilean outbreak. Biol Res 2002; 35: 433-40.

12.- Farmer III J J, Janda M, Birkhead K. En Murray P, Baron E J, Jörgensen J H, Pfaller M A, Yolken R H, eds. Manual of Clinical Microbiology 2003, $8^{\text {th }}$ Ed. ASM Press, Washington D.C., USA. p: 706-18.

13.- Peterson K M, Zuppardo A. Vibrio parahaemolyticus and Vibrio vulnificus. En Sussman M, ed. Molecular Medical Microbiology. 2002. Academic Press. $1^{\text {st }}$ Ed 2002, London, U.K. p: 1291-1309.

14.- Nishibuchi M, Kaper J B. Thermostable direct hemolysin gene of Vibrio parahaemolyticus: a virulence gene acquired by a marine bacterium. Infect Immun 1995; 63: 2093-9.

15.- Nishibuchi M, Kumagai K, Kaper J B. Contribution of the $t d h 1$ gene of Kanagawa phenomenon-positive Vibrio parahaemolyticus to production of extracellular thermostable direct hemolysin. Microb Pathog 1991; 11:453-60.

16.- Shirai H, Ito H, Hirayama T, Nakamoto Y, Nakaba- yashi N, Kumagai K, et al. Molecular epidemiologic evidence for association of thermostable direct hemolysin (TDH) and TDH-related hemolysin of Vibrio parahaemolyticus with gastroenteritis. Infect Immun 1990; 58: 3568-73.

17.- Kishishita M, Matsuoka N, Kumagai K, Yamasaki S, Takeda Y, Nishibuchi M. Sequence variation in the thermostable direct hemolysin-related hemolysin (trh) gene of Vibrio parahaemolyticus. Appl Environ Microbiol 1992; 58: 2449-57.

18.- Okuda J, Ishibashi M, Abbott $S$ L, Janda J M, Nishibuchi M. Analysis of the thermostable direct hemolysin $(t d h)$ gene and the tdh-related hemolysin (trh) genes in urease-positive strains of Vibrio parahaemolyticus isolated on the West Coast of the United States. J Clin Microbiol 1997; 35: 1965-71.

19.- Iida T, Park K S, Suthienkul O, Kozawa J, Yamaichi Y, Yamamoto K, et al. Close proximity of the $t d h, t r h$ and ure genes on the chromosome of Vibrio parahaemolyticus. Microbiology 1998; 144: 2517-23.

20.- Akeda Y, Kodama T, Kashimoto T, Cantarelli V, Horiguchi Y, Nagayama K, et al. Dominant-negative Rho,Rac, and Cds42 facilitate the invasion process of Vibrio parahaemolyticus into CaCO-2 cells. Infect Immun 2002; 70(2): 970-3.

21.- Iguchi T, Kondo S, Hisatsune K. Vibrio parahaemolyticus $\mathrm{O}$ serotypes from $\mathrm{O} 1$ to $\mathrm{O} 13$ all produce $\mathrm{R}$ type lipopolosaccharide: SDS PAGE and compositional sugar analysis. FEMS Microbiol Lett 1995; 130: 287 92.

22.- Elliot EL, Kaysner C A, Jackson L, Tamplin M L. Vibrio cholerae, V. parahaemolyticus, V. vulnificus, and other Vibrio spp., 1998. p. 9.01-9.27. US Food and Drug Administration Bacteriological Analytical Manual. A.O.A.C. International, Gaithersburg, Md. USA.

23.- Suthienkul O, Ishibashi M, Iida T, Nettip N, Supavej S, Eampokalap B, et al. Urease production correlates with possession of the trh gene in Vibrio parahaemolyticus strains isolated in Thailand. $\mathrm{J}$ Infect Dis 1995 ; 172: 1405-8.

24.- Marshall S, Clark CG, Wang G, Mulvey M, Kelly M T, Johnson W M. Comparison of molecular methods for typing Vibrio parahaemolyticus. J Clin Microbiol 1999; 37: 2473-8.

25.- Wong H C, Lu K T, Pan T M, Lee C L, Shih D Y. Subspecies typing of Vibrio parahaemolyticus by pulsed-field gel electrophoresis. J Clin Microbiol 1996; 34: $1535-9$.

26.- Chowdhury N R, Chakraborty S, Ramamurthy T, Nishibuchi M, Yamasaki S, Takeda, et al. Molecular evidence of clonal Vibrio parahaemolyticus pandemic strains. Emerg Infect Dis 2000; 6: 631-6.

27.- Wong H C. Detecting and molecular typing of Vibrio parahaemolyticus. J Food Drug Analysis 2003; 2: 7986.

28.- Matsumoto C, Okuda J, Ishibashi M, Iwanaga M, Garg P, Rammamurthy T, et al. Pandemic spread of an O3:K6 clone of Vibrio parahaemolyticus and emergence of related strains evidenced by arbitrarily primed PCR and toxRS sequence analyses. J Clin Microbiol 2000; 38: 578-85.

29.- Okuda J, Ishibashi M, Hayakawa E, Nishino T, Takeda Y, Mukhopadhyay A K, et al. Emergence of a unique O3:K6 clone of Vibrio parahaemolyticus in Calcutta, India, and isolation of strains from the same clonal group from Southeast Asian travelers arriving in Japan. J Clin Microbiol 1997; 35: 3150-5. 
30.- Wong H C, Lin C H. Evaluation of typing of Vibrio parahaemolyticus by three PCR methods using specific primers. J Clin Microbiol 2001; 39: 4233-40.

31.- Chowdhury N R, Stine O C, Morris J G, Nair G B. Assessment of evolution of pandemic Vibrio parahaemolyticus by multilocus sequence typing. J Clin Microbiol 2004; 42: 1280-2.

32.- DePaola A, Ulaszek J, Kaysner C A, Tenge B J, Nordstrom J L, Wells J, et al. Molecular, serological, and virulence characteristics of Vibrio parahaemolyticus isolated from environmental, food, and clinical sources in North America and Asia. Appl Environ Microbiol 2003; 69: 3999-4005.

33.- Bhuiyan N A, Ansaruzzaman M, Kamruzzaman M, Alam K, Chowdhury N R, Nishibuchi M, et al. Prevalence of the pandemic genotype of Vibrio parahaemolyticus in Dhaka, Bangladesh, and significance of its distribution across different serotypes. J Clin Microbiol 2002; 40: 284-6.

34.- Hara-Kudo Y, Sugiyama K, Nishibuchi M, Chowdhury A, Yatsuyanagi J, Ohtomo Y, et al. Prevalence of pandemic thermostable direct hemolysin-producing Vibrio parahaemolyticus O3:K6 in seafood and the coastal environment in Japan. Appl Environ Microbiol 2003; 69: 3883-91.

35.- González-Escalona N, Cachicas V, Acevedo C, Rioseco M L, Vergara J A, Cabello F, et al. Vibrio parahaemolyticus diarrhea, Chile, 1998 and 2004. Emerg Infect Dis 2005; 11: 129-31.

36.- Yi-Shin Chen,Meng-Yi Chen, Hin-Chung Wong, Susceptibility of O:K6 and environmental strains of Vibrio parahaemolyticus to acid inactivation, and survival competition between these strains and Pseudomonas fluorescens and indigenous bacteria. J Food Drug Analysis 2003; 11: 226-32.

37.- Islam M S, Tasmin R, Khan S I, Bakht H B, Mahmood Z H, Rahman M Z, et al. Pandemic strains of O3:K6 Vibrio parahaemolyticus in the aquatic environment of Bangladesh. Can J Microbiol/Rev Can
Microbiol 2004; 50: 827-34.

38.- Wong H C, Liu S H, Wang T K, Lee C L, Chiou C S, Liu D P, et al. Characteristic of Vibrio parahaemolyticus O3:K6 from Asia. Appl Environ Microbiol 2000; 66: 3981-6.

39.- Lesmana M, Subekti D, Simanjuntak C H, Tjaniadi P, Campbell J R, Oyofo B A. Vibrio parahaemolyticus associated with cholera like diarrhrea among patients in North Jakarta, Indonesia. Diag Microbiol Infect Dis 2000; 39: 71-5.

40.- Khamet A L, Tabrez S, Link R, Roberts I. Vibrio parahaemolyticus as a cause for necrotizing fasciitis in a patient with cirrhosis. Am J Gastroenterol 2001: S125.

41.- Ruiz C, Agraharkar M. Inusual marine pathogens causing cellulitis and bacteremia in hemodialysis patients: report of the three cases and review of the literature. Hemodial Int 2003; 7: 356-9.

42.- Matsumoto C S, Fishbein T M, Kaufman S. Gastrointestinal infections in solid organ transplant recipients. Curr Opin Organ Transplant 2004, 9: 406-10.

43.- Shankar V K, Zilvetti M, Handa A, Bowler I C, Gray D W. Chronic diarrhea and weight loss due to Vibrio parahaemolyticus infection in a renal transplant recipient. Transplantation 2004; 78: 487.

44.- Ho H, Amin H. E-medicine: Vibrio Infections. 2000; http://www.emedicine.com/med/topic2375.htm. (accedido 10 marzo 2005)

45.- Tang H J, Chang M C, Ko W C, Huang K Y, Lee C L, Chuang Y C. In vitro and in vivo activities of newer fluoroquinolones against Vibrio vulnificus. Antimicrob Agents Chemother 2002; 46: 3580-4.

46.- DePaola A, Nordstrom J L, Bowers J C, Wells J G, Cook D W. Seasonal abundance of total and pathogenic $V$. parahaemolyticus in Alabama oysters. Appl Environ Microbiol 2003; 69: 1521-6.

47.- Huang K C, Hsu R W. Vibrio fluvialis hemorragic cellulitis and cerebritis. Clin Infect Dis 2005; 40: e 75-7.

Correspondencia a:

Ingrid Heitmann Giglioto

iheitmann@isp.cl 